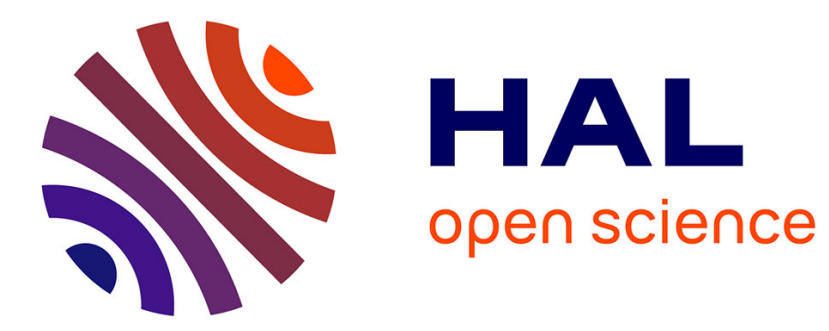

\title{
Dynamical analysis of multi-stage cyclic structures
}

Denis Laxalde, Fabrice Thouverez, Jean-Pierre Lombard

\section{To cite this version:}

Denis Laxalde, Fabrice Thouverez, Jean-Pierre Lombard. Dynamical analysis of multistage cyclic structures. Mechanics Research Communications, 2007, 34 (4), pp.379-384. 10.1016/j.mechrescom.2007.02.004 . hal-00227538

\section{HAL Id: hal-00227538 \\ https://hal.science/hal-00227538}

Submitted on 31 Jan 2008

HAL is a multi-disciplinary open access archive for the deposit and dissemination of scientific research documents, whether they are published or not. The documents may come from teaching and research institutions in France or abroad, or from public or private research centers.
L'archive ouverte pluridisciplinaire HAL, est destinée au dépôt et à la diffusion de documents scientifiques de niveau recherche, publiés ou non, émanant des établissements d'enseignement et de recherche français ou étrangers, des laboratoires publics ou privés. 
Journal home page: http://www.sciencedirect.com/science/journal/00936413

Dynamical Analysis of Multi-Stage Cyclic Structures

Mechanics Research Communications, Volume 34, Issue 4, June 2007, Pages 379-384

D. Laxalde, F. Thouverez, and J.-P. Lombard

\title{
Dynamical Analysis of Multi-Stage Cyclic Structures
}

\author{
D. Laxalde ${ }^{a, b}$, F. Thouverez ${ }^{a}$ and J.-P. Lombard ${ }^{b}$ \\ (a) Laboratoire de Tribologie et Dynamique des Systèmes (UMR CNRS 5513) \\ École Centrale de Lyon, 36 avenue Guy de Collongue, 69134 Ecully Cedex, France \\ (b) Snecma - Safran group, 77550 Moissy-Cramayel, France
}

\begin{abstract}
This paper deals with the dynamical analysis of a multi-stage assembly of cyclic structures such as, for example, turbomachinery compressor or turbines. If such assemblies are traditionally modelled stage by stage, the inter-stage coupling effect can sometime be important. As an answer to this issue, we propose a new method which combines a cyclic modelling of each stage with a realistic inter-stage coupling. Study cases are presented to evaluate the efficiency of the method.
\end{abstract}

Keywords: multi-stage, bladed disk, cyclic symmetry, modal analysis

\section{Introduction}

Rotationally periodic structures are used in many engineering applications such as turbine bladed disks or gear wheels. It is well known that the dynamics of such structures can be studied using the cyclic symmetry reduction. This method makes it possible to analyse the complete cyclic structure by considering a single elementary sector. Examples in the literature are numerous on this subject, from the early developments of cyclic symmetry method Thomas, 1979, Wildheim, 1981] to specific studies on bladed disks dynamics Srinivasan, 1984, Slater, 1999]. Also, even if some applications involve an assembly of cyclic structures leading to a multi-stage pattern (such as turbines or compressors), most existing studies focus on single stage analysis and the influence of the interstage coupling is usually neglected. Nevertheless, in some cases, this coupling can modify noticeably the dynamics of the structures; Bladh et al. 2003] have, for example, shown that the dynamics of the multi-stage assembly significantly differed from the single stage predictions (underestimation of vibratory levels).

Concerning modelling aspects, the major issue is that the cyclic symmetry reduction cannot be applied directly to the multi-stage assembly. Rzadkowski and Drewczynski [2004, 2006] have used full 360 models to study the (free and forced) dynamics of multi-stage systems. However, as such techniques often yield prohibitive computational costs, the use of component mode synthesis methods (with multilevel reductions) have been proposed [Bladh et al., 2001]. These strategies are efficient but can be somewhat difficult to use in a design process. In the present paper, we put forward a new strategy to study the dynamics of multi-stage assemblies using a cyclic symmetry reduction. This method handles the sector finite element modelling directly and involves a realistic interstage coupling. Following a brief review of the cyclic symmetry method (Section 2), the proposed method will be detailed in Section 3. Then, some numerical example and results will be presented in Section 4 . 


\section{Cyclic symmetry for single stages}

Let's consider a cyclic structure composed of $N$ sectors; as a consequence of the rotational periodicity, the mode shapes are such that each sector has the same deflection and a constant phase difference from the adjacent sectors. This inter-sector phase angle is $\beta_{n}=2 \pi n / N$ and the harmonic index (or wave number) $n=0,1, \ldots, N-1$ is called the nodal diameter number. The dynamical problem of the complete cyclic structure can be transform (exactly) into $N$ reduced problems (corresponding to each nodal diameter number $n$ ) on the reference sector with appropriate inter-sector boundary conditions. Then, if the reference sector displacement vector $\boldsymbol{u}_{0}$ is broken down into left ${ }^{l} \boldsymbol{u}_{0}$ and right $^{r} \boldsymbol{u}_{0}$ inter-sector degrees of freedom, we have, for the $n^{\text {th }}$ nodal diameter number:

$$
{ }^{l} \boldsymbol{u}_{0}=e^{-\frac{2 i \pi n}{N} r} \boldsymbol{u}_{0}
$$

A displacement vector, in the cyclic base, $\tilde{\boldsymbol{u}}_{n}$ can be defined by eliminating the left part of reference sector degrees of freedom $\boldsymbol{u}_{0}$ for nodal diameter number $n$. Finally, introducing the cyclic constraints given by equation (11) in the structural matrices of the reference sector an eigenvalue equation, for the $n$-th nodal diameter is obtained:

$$
K_{n} \tilde{\boldsymbol{u}}_{n}=M_{n} \tilde{\boldsymbol{u}}_{n} \lambda
$$

Each reduced problem (2) is solved in cyclic coordinates $\tilde{\boldsymbol{u}}_{n}$ (for the reference sector) and the transformation in physical coordinates (complete structure) is given by:

$$
\boldsymbol{u}=\boldsymbol{e}_{n} \otimes \tilde{\boldsymbol{u}}_{n} \text { with } \boldsymbol{e}_{n}=\left[\begin{array}{lllll}
1 & e^{\frac{2 i \pi n}{N}} & e^{\frac{4 i \pi n}{N}} & \ldots & e^{\frac{2(N-1) i \pi n}{N}}
\end{array}\right]^{T}
$$

where $\otimes$ is the Kronecker product.

Other dynamical problems (forced or transient responses for example) can also be solve using this method. For further readings, see references [Thomas, 1979, Wildheim, 1981, Henry, 1980].

\section{A multi-stage cyclic symmetry method}

We suggest a new strategy to analyse the dynamics of multi-stage cyclic structures. It is called multi-stage cyclic symmetry since the cyclic symmetry strategy is used to model each stage. The interstage coupling is ensured through a cyclic expansion of the interface degrees of freedom of each stages. The method will be detailed based on the example of two stages respectively composed of $N_{1}$ and $N_{2}$ sectors $\left(N_{1} \neq N_{2}\right)$. The method is presented assuming a mesh compatibility of the interstage interfaces. However, in cases where the interstage interfaces would not match the use of multi-point constraints can be a solution but this does not restrict the present method.

For the $(i)$-th stage $(i=1,2)$, we define the vector containing the degrees of freedom of the reference sector in the appropriate cyclic base for the $n$-th nodal diameter number $\tilde{\boldsymbol{u}}_{n}^{(i)}$ with the notations defined in Section 2. We also define, in physical and cyclic bases, ${ }^{b} \boldsymbol{u}^{(i)}$ and ${ }^{b} \tilde{\boldsymbol{u}}_{n}^{(i)}$ the restriction of the degrees of freedom vectors to the interstage degrees of freedom and, ${ }^{o} \boldsymbol{u}^{(i)}$ and ${ }^{o} \tilde{\boldsymbol{u}}_{n}^{(i)}$ those related to other degrees of freedom. We additionally introduce identity matrices $\mathcal{I}_{b_{i}}$ and $\mathcal{I}_{o_{i}}$ which size correspond to the number of boundary and internal degrees of freedom respectively of the $i$-th stage.

The interstage compatibility can be ensured in the (common) physical base

$$
{ }^{b} \boldsymbol{u}^{(1)}={ }^{b} \boldsymbol{u}^{(2)}
$$


and since for each interface, we have,

$$
{ }^{b} \boldsymbol{u}^{(1)}=\left(\boldsymbol{e}_{N_{1}, n} \otimes \mathcal{I}_{b_{1}}\right){ }^{b} \tilde{\boldsymbol{u}}_{n}^{(1)} \text { and }{ }^{b} \boldsymbol{u}^{(2)}=\left(\boldsymbol{e}_{N_{2}, n} \otimes \mathcal{I}_{b_{2}}\right){ }^{b} \tilde{\boldsymbol{u}}_{n}^{(2)}
$$

equation (44) can be rewritten in terms of cyclic coordinates as,

$$
{ }^{b} \tilde{\boldsymbol{u}}_{n}^{(2)}=\mathcal{B}_{n}{ }^{b} \tilde{\boldsymbol{u}}_{n}^{(1)} \text { with } \mathcal{B}_{n}=\left(\boldsymbol{e}_{N_{2}, n}^{*} \otimes \mathcal{I}_{b_{2}}\right)\left(\boldsymbol{e}_{N_{1}, n} \otimes \mathcal{I}_{b_{1}}\right)
$$

The matrix $\mathcal{B}_{n}$ is the projection matrix of the $n$-th cyclic vector of stage 1 on the $n$-th cyclic vector of stage 2 . We can then perform the assembly of the two stages degrees of freedom vector

$$
\left[\begin{array}{l}
\tilde{\boldsymbol{u}}_{n}^{(1)} \\
\tilde{\boldsymbol{u}}_{n}^{(2)}
\end{array}\right]=\mathcal{T}_{n} \tilde{\boldsymbol{u}}_{n}^{(1 \cup 2)} \text { with, } \mathcal{T}_{n}=\left(\begin{array}{ccc}
\mathcal{I}_{b_{1}} & 0 & 0 \\
0 & \mathcal{I}_{o_{1}} & 0 \\
B_{n} & 0 & 0 \\
0 & 0 & \mathcal{I}_{o_{2}}
\end{array}\right) \text { and } \tilde{\boldsymbol{u}}_{n}^{(1 \cup 2)}=\left[\begin{array}{l}
{ }^{b} \tilde{\boldsymbol{u}}_{n}^{(1)} \\
{ }^{o} \tilde{\boldsymbol{u}}_{n}^{(1)} \\
{ }^{o} \tilde{\boldsymbol{u}}_{n}^{(2)}
\end{array}\right]
$$

and define the two stages eigenvalue problem:

$$
K_{n}^{(1 \cup 2)} \tilde{\boldsymbol{u}}_{n}^{(1 \cup 2)}=M_{n}^{(1 \cup 2)} \tilde{\boldsymbol{u}}_{n}^{(1 \cup 2)} \lambda
$$

with,

$$
K_{n}^{(1 \cup 2)}=\mathcal{T}_{n}^{T}\left(\begin{array}{cc}
K_{n}^{(1)} & 0 \\
0 & K_{n}^{(2)}
\end{array}\right) \mathcal{T}_{n} \text { and } M_{n}^{(1 \cup 2)}=\mathcal{T}_{n}^{T}\left(\begin{array}{cc}
M_{n}^{(1)} & 0 \\
0 & M_{n}^{(2)}
\end{array}\right) \mathcal{T}_{n}
$$

This method enables the study of nodal diameter modes separately as for individual stages, each being modelled with its own elementary sector. The size of problem (8) is close to the sum of the two individual stages problem sizes. The "nodal diameter modes" found with the present method are not truly modes of the multi-stage system since it is not cyclic; however, the numerical results (Section 4) will show that they are a good set of basis vectors and provide a very good approximation to system's dynamics.

\section{Numerical results}

In this section we evaluate the performance of the multi-stage cyclic symmetry method on a numerical example. We consider two stages of cyclic structures with simple geometry; the first being composed of 24 elementary sectors and the second of 45 elementary sectors. The figure 1 shows the finite elements meshes of the two elementary sectors and of the whole structure. First we have compared the results on the multi-stage cyclic symmetry method to a full 360 analysis taken as a reference. In figure 2, the eigenfrequencies calculated by the two methods plotted versus their nodal diameter index are displayed. The correlation appears to be correct since the eigenfrequency deviation between the two methods is very small. Also note that all modes are properly captured (none are missed) with multi-stage cyclic symmetry method; this attests the quality of the "cyclic condition" approximation of the method.

Next, we show and compare (in a qualitative way) the modal deformed shapes. Figures 3 and 4 show results from (a) the reference analysis and (b) the multi-stage cyclic symmetry for various nodal diameter numbers. In these examples we can distinguish some modes localized in only one stage (figure 4) and some others with a clear multi-stage behaviour (figure 3).

Finally, we found interesting to show the influence of the interstage coupling by comparing the results of single stage analysis to the multi-stage case. For the single stage analysis we applied a fixed boundary condition on the interstage interface. The figure 5 gathers the eigenfrequencies of these three calculations. The influence of the interstage coupling appears clearly and new 

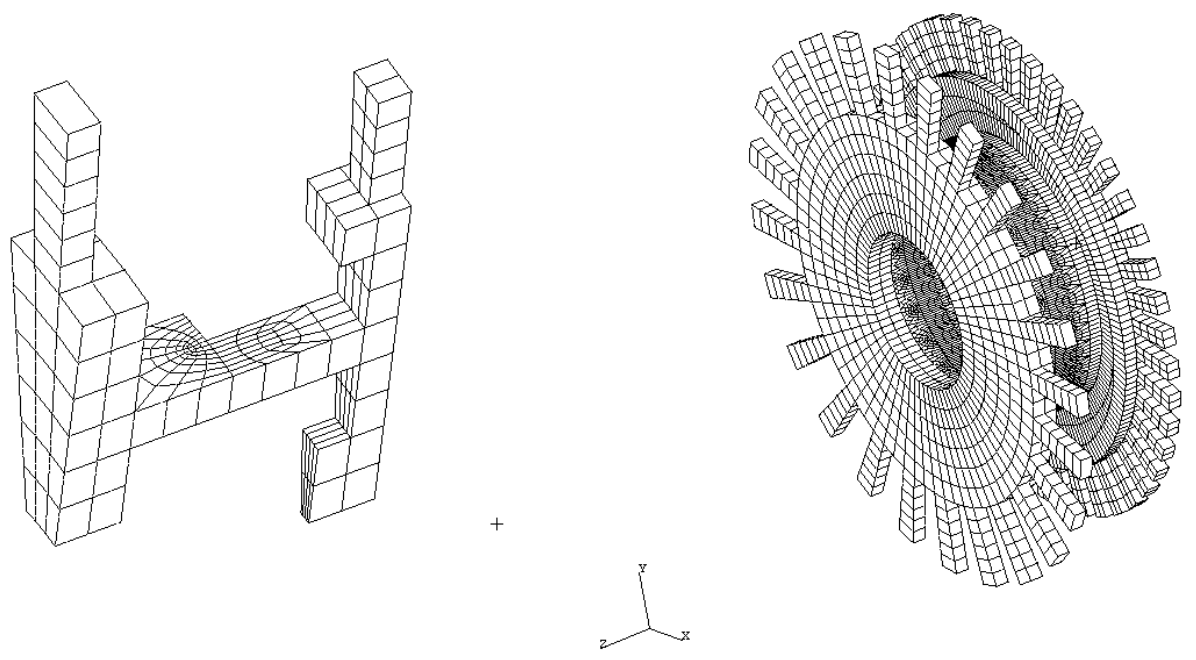

Figure 1: Finite element model of 2 cyclic structures $-N_{1}=24, N_{2}=45$

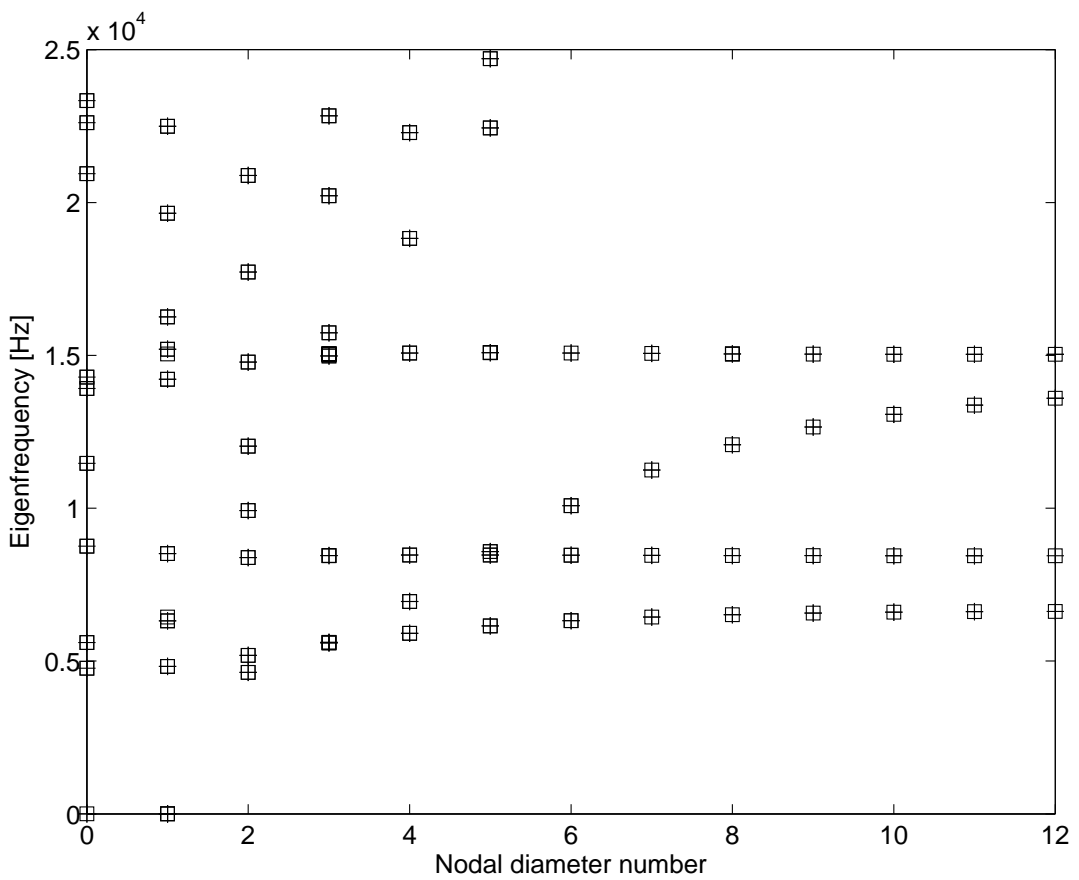

Figure 2: Eigenfrequency correlation: Eigenfrequency/nodal diameter map, reference $\square$ / multistage cyclic symmetry + . 


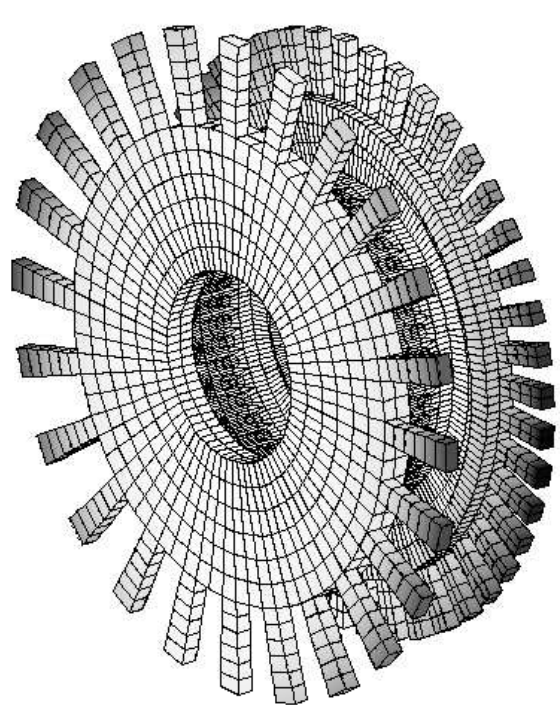

(a)

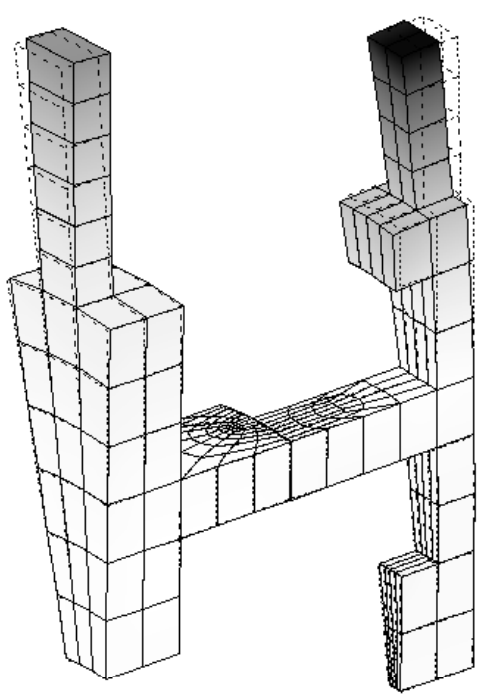

(b)

Figure 3: One nodal diameter mode at $4824 \mathrm{~Hz}$

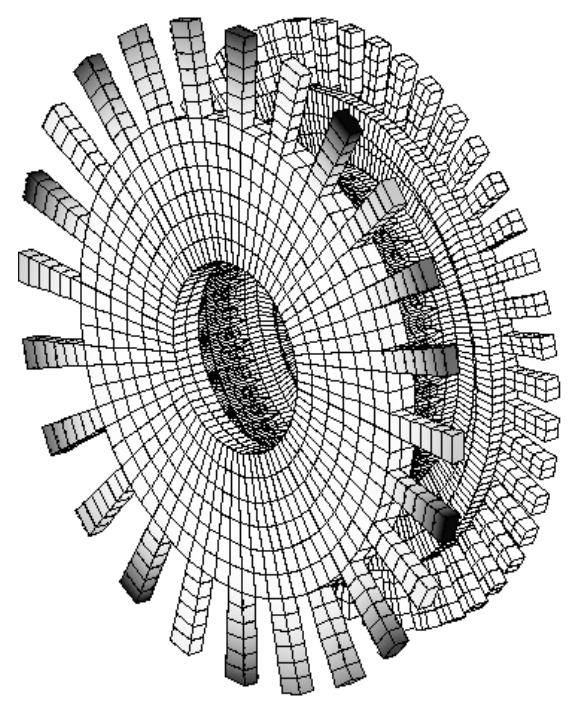

(a)

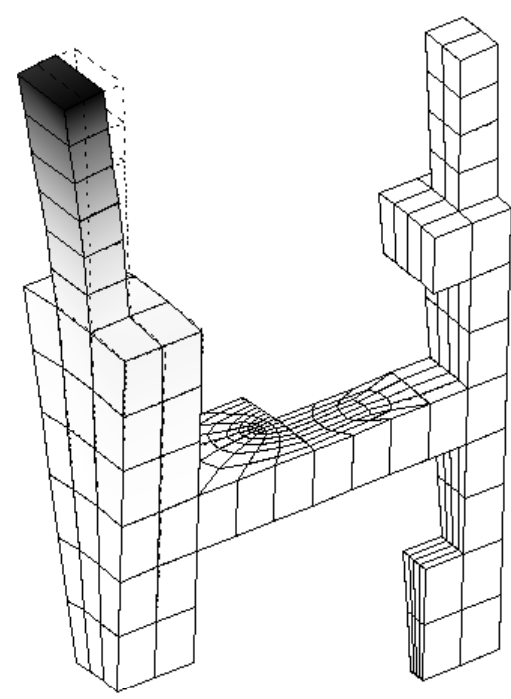

(b)

Figure 4: Five nodal diameter mode at $6147 \mathrm{~Hz}$ 


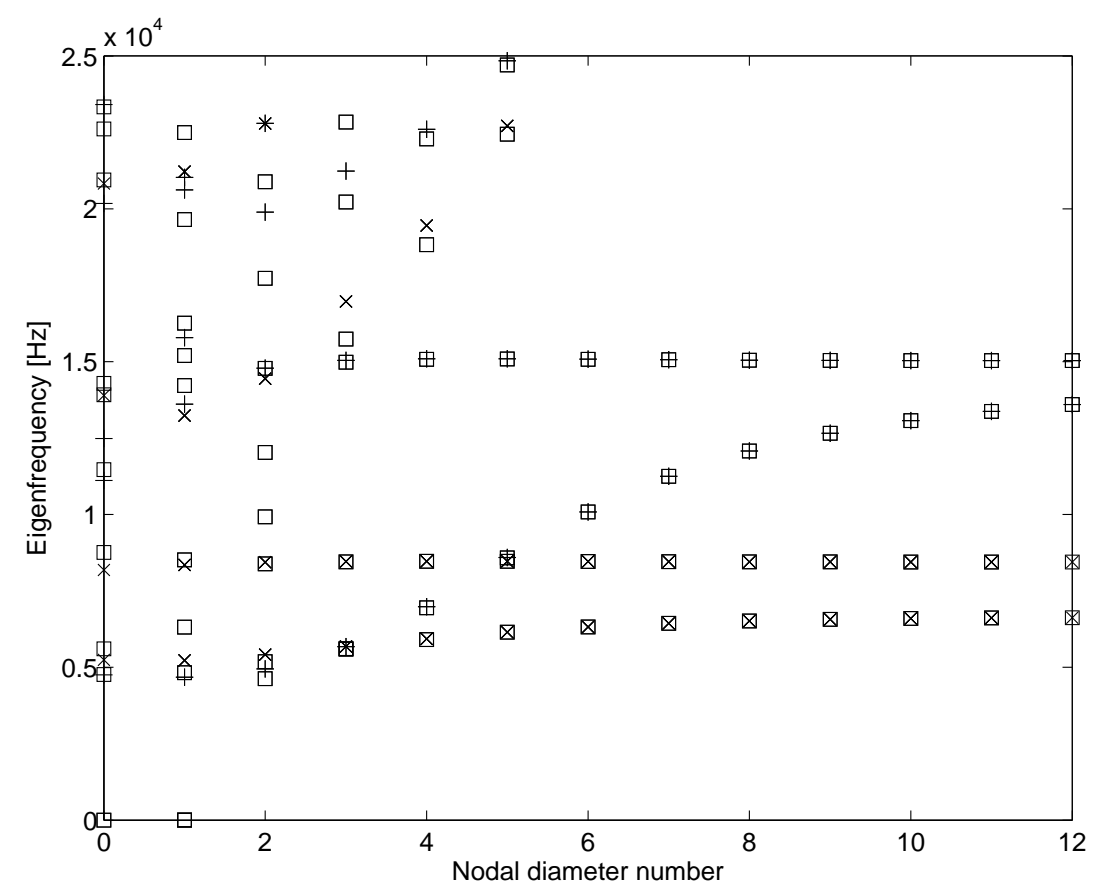

Figure 5: Influence of interstage coupling: Eigenfrequency/nodal diameter map, single stages (stage $\# 1 \times$ and stage $\# 2+$ ) / coupled stages $\square$.

modes appears that could not be predicted by a single stage analysis. This is particularly clear in small nodal diameter numbers. In effect, in these cases, the blade/disk coupling is generally more important than for higher nodal diameter numbers where the disk's participation is usually smaller. This comparison clearly shows the influence of the multi-stage coupling in the blade disks design.

\section{Conclusions}

A new strategy to study the dynamics of multi-stage cyclic structures have been proposed. It is based on a cyclic symmetry modelling of individual stages (in their respective cyclic base) and an accurate interstage coupling in the common physical base. This method appears quite simple to carry out since the sector models of each stage are handled directly and the coupling involves few additional difficulties. The results from numerical simulations show a good accuracy of this method.

\section{References}

R. Bladh, M. P. Castanier, and C. Pierre. Reduced order modeling techniques for dynamic analysis of mistuned multi-stage turbomachinery rotors. In Proceedings of ASME Turbo Expo, number 2001- GT-0276, New Orleans, LA, USA, 2001.

R. Bladh, M. P. Castanier, and C. Pierre. Effects of multistage coupling and disk flexibility on mistuned bladed disk dynamics. Journal of Engineering for Gas Turbines and Power, 125: 121-130, 2003. 
R. Henry. Calcul des fréquences et modes des structures répétitives circulaires. Journal de Mécanique appliquée, 4(1):61-82, 1980.

R. Rzadkowski and M. Drewczynski. Natural frequencies and modes shapes of two mistuned bladed discs on the shaft. In Proceedings of ASME Turbo Expo, number GT2004-54265, Vienna, Austria, 2004.

R. Rzadkowski and M. Drewczynski. Forced vibration of several bladed discs on the shaft. In Proceedings of ASME Turbo Expo, number GT2006-90158, Barcelona, Spain, 2006.

J. C. Slater. Forced response of bladed disks assemblies - a survey. The shock and vibration digest, 31(1):17-24, January 1999.

A. V. Srinivasan. Vibrations of bladed disks assemblies - a selected survey. Journal of Vibration, Acoustics, Stress, and Reliability in Design, 106, 1984.

D. L. Thomas. Dynamics of rotationally periodic structures. International Journal for Numerical Methods in Engineering, 14:81-102, 1979.

J. Wildheim. Excitation of rotationally circumferentially periodic structures. Journal of Sound and Vibration, 75(3):397-416, 1981. 